\title{
Dual-gratings with a Bragg reflector for dielectric laser- driven accelerators
}

DOI:

$10.1063 / 1.4993206$

Document Version

Accepted author manuscript

Link to publication record in Manchester Research Explorer

\section{Citation for published version (APA):}

Wei, Y., Xia, G., Smith, J. D. A., \& Welsch, C. P. (2017). Dual-gratings with a Bragg reflector for dielectric laserdriven accelerators. Physics of Plasmas, 24(7), [073115]. https://doi.org/10.1063/1.4993206

\section{Published in:}

Physics of Plasmas

\section{Citing this paper}

Please note that where the full-text provided on Manchester Research Explorer is the Author Accepted Manuscript or Proof version this may differ from the final Published version. If citing, it is advised that you check and use the publisher's definitive version.

\section{General rights}

Copyright and moral rights for the publications made accessible in the Research Explorer are retained by the authors and/or other copyright owners and it is a condition of accessing publications that users recognise and abide by the legal requirements associated with these rights.

\section{Takedown policy}

If you believe that this document breaches copyright please refer to the University of Manchester's Takedown Procedures [http://man.ac.uk/04Y6Bo] or contact uml.scholarlycommunications@manchester.ac.uk providing relevant details, so we can investigate your claim.

\section{OPEN ACCESS}




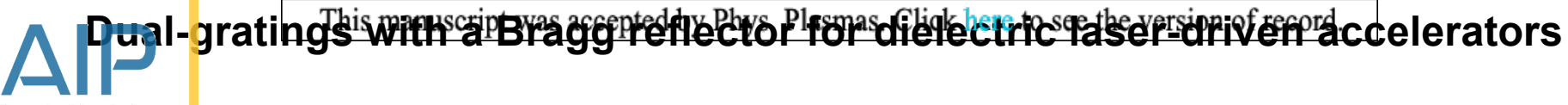 \\ Y. Wei, ${ }^{1,2, a)}$ G. Xia, ${ }^{1,3}$ J. D. A. Smith, ${ }^{4}$ and C.P. Welsch, ${ }^{1,2}$}

${ }^{1}$ Cockcroft Institute, Sci-Tech Daresbury, Warrington, WA4 4AD, United Kingdom

${ }^{2}$ Physics Department, University of Liverpool, Liverpool, L69 3BX, United Kingdom

${ }^{3}$ School of Physics and Astronomy, University of Manchester, Manchester, M13 9PL, United Kingdom

${ }^{4}$ Tech-X UK Ltd, Sci-Tech Daresbury, Warrington, WA4 4AD, United Kingdom

The acceleration of a beam of electrons has been observed in a dielectric laser-driven accelerator (DLA) with a gradient of $300 \mathrm{MV} / \mathrm{m}$. It opens the way to building a particle accelerator "on a chip" much more cheaply than a conventional one. This paper investigates numerically an efficient dielectric laser-driven accelerating structure, based on dual-gratings with a Bragg reflector. The design of the structure boosts the accelerating field in the channel, thereby increasing the accelerating gradient by more than $70 \%$ compared to bare dual-gratings, from analytical calculations. This is supported by two-dimensional (2D) particle-in-cell simulations, where a $50 \mathrm{MeV}$ electron bunch is loaded into an optimized 100-period structure to interact with a $100 \mathrm{fs}$ pulsed laser having a peak field of $2 \mathrm{GV} / \mathrm{m}$. It demonstrates a loaded accelerating gradient of $1.48 \pm 0.10 \mathrm{GV} / \mathrm{m}$, which is $(85 \pm 26) \%$ higher than that of bare dual-gratings. In addition, studies of the diffraction effect show that the optimized structure should be fabricated with a vertical size of $J / w_{\mathrm{x}} \geq 0.20$ in order to generate an acceptable accelerating performance.

\section{Introduction}

The rapid development of laser technology, combined with mature fabrication techniques for microstructures, has enabled the novel accelerating scheme of dielectric laser-driven accelerators (DLAs) to be developed. This novel technique employs laser light to propel electrons through a microscopic tunnel in artfully crafted glass chips, and has the potential to dramatically shrink the size and cost of particle accelerators. Many DLAs have been proposed, with specific designs: gratingbased structures ${ }^{1-4}$, photonic band-gap structures ${ }^{5-7}$ and woodpile structures ${ }^{8-10}$. Recent DLA experiments have demonstrated accelerating gradients of $300 \mathrm{MV} / \mathrm{m}^{11}, 690 \mathrm{MV} / \mathrm{m}^{12}$ for relativistic electrons and gradients of $25 \mathrm{MV} / \mathrm{m}^{13}, 220 \mathrm{MV} / \mathrm{m}^{14}$ and $370 \mathrm{MV} / \mathrm{m}^{15}$ for non-relativistic electrons. These demonstrations pave the way for implementing a new generation of accelerators "on a chip" in the future.

The dual-grating structures proposed by Plettner et al. ${ }^{1}$ have a simpler geometry than other types of DLAs, which reduces the complexity and expense of the fabrication process. They can be illuminated by a single laser beam to excite spatial harmonics modes in the narrow channel gap where the electrons travel and are accelerated. The grating period $\lambda_{\mathrm{p}}$, laser wavelength $\lambda_{0}$ and electron velocity $v, \beta=v / c$ have to meet the synchronicity condition ${ }^{16}: \lambda_{\mathrm{p}}=m \beta \lambda_{0}$ where $m$ is the number

\footnotetext{
a) Electronic mail: yelong.wei@cockcroft.ac.uk.
} 

out for the dual-grating structures. A Bragg reflector consisting of many layers of Sapphire was also proposed for dualgratings with the same material, which could generate an accelerating gradient as much as double the incident laser field ${ }^{17}$. However, fabrication techniques for Sapphire are not as well developed as for the quartz material, which has been fabricated and demonstrated in many DLA experiments ${ }^{11-13}$. In addition, when the fabricated vertical size $J$, as shown in Fig. 1, is smaller than the transverse laser waist radius, the resulting diffraction may substantially reduce the accelerating gradient, an effect which has not been studied in Ref. 17.

Motivated by these two points, we present in this paper detailed numerical studies of a quartz DLA structure based on dual-gratings with a Bragg reflector, including analytical optimizations, particle-in-cell simulations and diffraction effect studies. As shown in Fig. 1, a Bragg reflector reflects back laser power to enhance the accelerating field in the channel, thereby increasing the accelerating gradient for electrons. Compared to the dual-grating structures reported in Refs 1-4, it has the extra geometry parameter of distance $D$ between the dual-gratings and the Bragg reflector, as seen in Fig. 1. It can be optimized to generate the maximum accelerating field by creating constructive interference in the channel center. Moreover, mature lithographic techniques allow the dual-gratings to be integrated with a Bragg reflector into a single wafer, with nanometer precision and at low cost ${ }^{18,19}$. Section $\mathrm{H}$ presents detailed $2 \mathrm{D}$ analytical optimizations for such dual-gratings with a Bragg reflector. It is followed in Section III by 2D particle-in-cell simulations in which a $100 \mathrm{fs}$ pulsed laser is introduced into an optimized 100-period dual-grating structure to interact with a $50 \mathrm{MeV}$ electron bunch. Section IV then investigates the potential diffraction effect which is caused by a fabricated vertical size smaller than the transverse laser waist radius. Finally, the potential applications and challenges are discussed.

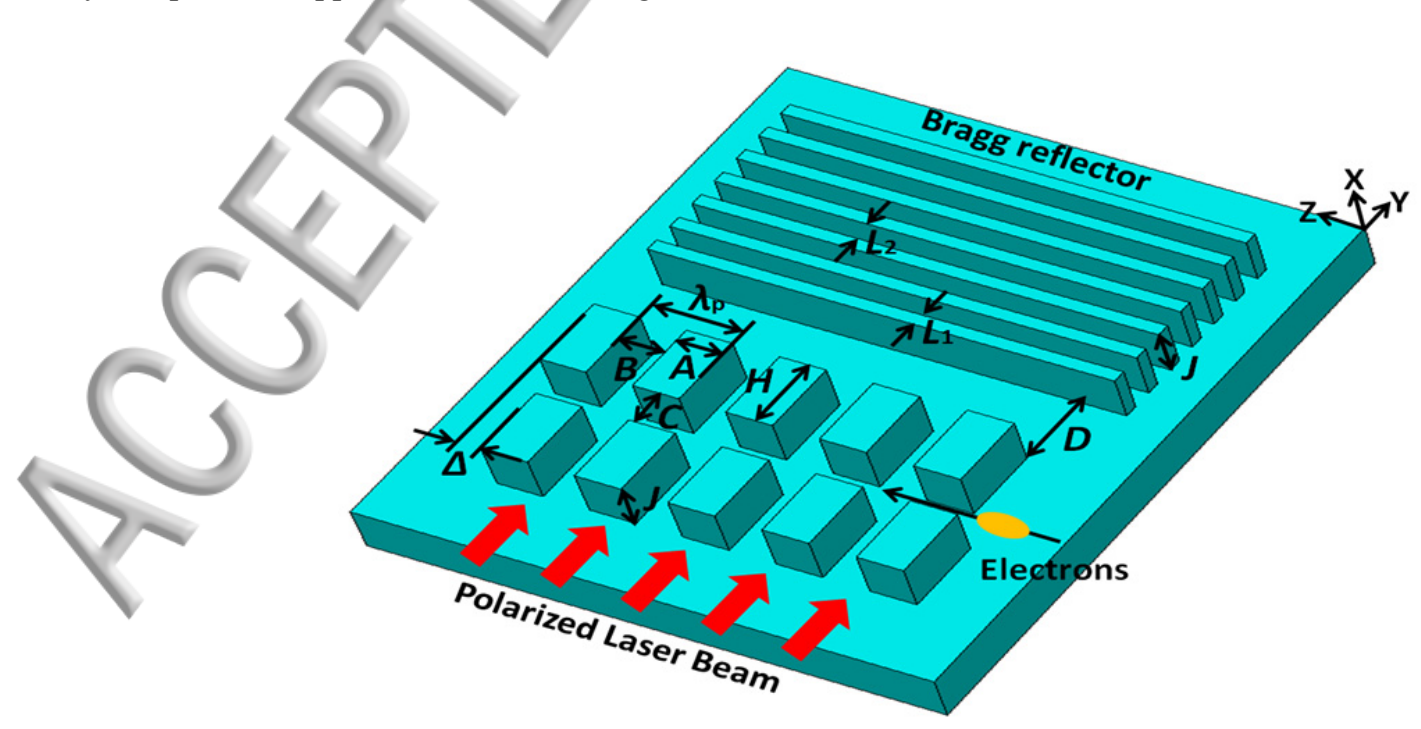




\section{2D Analytical Optimizations}

In order to simulate such a structure, the code $\mathrm{VSim}^{20}$, based on finite difference time domains (FDTD), is used to compute the electric and magnetic fields generated in the structure. It can also perform intensive particle-in-cell simulations to obtain all the relevant physical characteristics of the structures.

As a first step, we start by modelling a dual-grating structure without a Bragg reflector. As shown in Fig. 3(a), when a uniform plane wave travels through a single-period structure, the speed of wave in the vacuum is higher than that in the dielectric grating pillar. This creates a phase difference of $\pi$ for the wave front in the vacuum channel where electrons are travelling and are periodically modulated along the longitudinal $z$-axis. To produce the desired phase difference of $\pi$, the grating pillar height $H$ could be set to $H=\frac{\lambda_{0}}{2(n-1)}=\lambda_{0}$, where $n=1.5$ is the refractive index of the quartz chosen as the grating material. The grating period $\lambda_{\mathrm{p}}$ equals the laser wavelength $\lambda_{0}$, so that relativistic electrons are synchronous with the first spatial harmonics ${ }^{16}$, this being the most efficient compared to other spatial harmonics.

After setting the phase difference and synchronicity, we need to optimize the longitudinal electric field $E_{\mathrm{z}}[z(t), t]$ in the channel center to maximize the accelerating gradient $G$, which is defined as:

$$
G=\frac{1}{\lambda_{\mathrm{p}}} \int_{0}^{\lambda_{\mathrm{p}}} E_{\mathrm{z}}[\mathrm{z}(t), t] d z
$$

where $z(t)$ is the position of the electrons in the vacuum channel at time $t$. It should be noted here that two important factors are usually used to evaluate the accelerating performance: accelerating efficiency $A E=G / E_{0}$ and accelerating factor $A F=$ $G / E_{\mathrm{m}}$, where $E_{\mathrm{m}}$ is the maximum field distributed in the structure for an input laser field of $E_{0}$. When $E_{\mathrm{m}}$ is lower than the material damage threshold, optimizations are carried out to maximize the accelerating efficiency. However, when $E_{\mathrm{m}}$ is close to the material damage threshold, it is necessary to restrict the maximum field in the gratings, which should not exceed this threshold.

To find the optimum designs for the dual-gratings without a Bragg reflector, we vary $C, A, \Delta$ to maximize the accelerating factor or accelerating efficiency. Reported studies ${ }^{1}$ have shown that for such dual-gratings, the accelerating gradient usually decreases for a larger channel gap. A gap of $C=0.50 \lambda_{\mathrm{p}}$ is therefore chosen to start our optimization due to the trade-off between the accelerating gradient and the available phase space in which high gradient occurs. Then we sweep the pillar width $A$ from $0.10 \lambda_{\mathrm{p}}$ to $0.90 \lambda_{\mathrm{p}}$ combined with variable longitudinal shift $\Delta$ from $-0.50 \lambda_{\mathrm{p}}$ to $0.50 \lambda_{\mathrm{p}}$ for optimizations. Figure 2(a) shows that the maximum $A F=0.155$ can be obtained at $C=0.50 \lambda_{\mathrm{p}}, H=\lambda_{\mathrm{p}}, A=0.60 \lambda_{\mathrm{p}}, \Delta=0 \mathrm{~nm}$ while the 


\section{This manuscript was accepted by Phys. Plasmas. Click here to see the version of record.}

$A E=0.440$ occurs at $C=0.50 \lambda_{\mathrm{p}}, H=\lambda_{\mathrm{p}}, A=0.50 \lambda_{\mathrm{p}}, \Delta=0 \mathrm{~nm}$, which can be seen in Fig. 2(b). The dual-gratings Publisshfding best when they are perfectly aligned $(\Delta=0 \mathrm{~nm})$, which agrees well with the results from England et al. ${ }^{21}$.

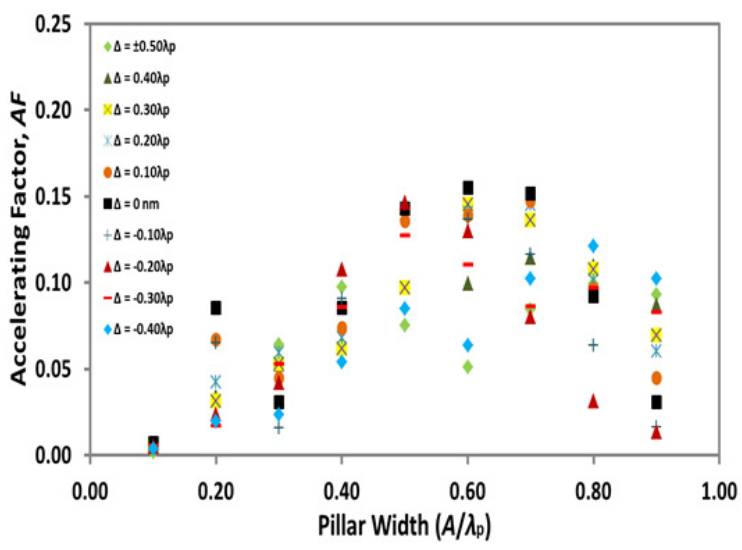

(a)

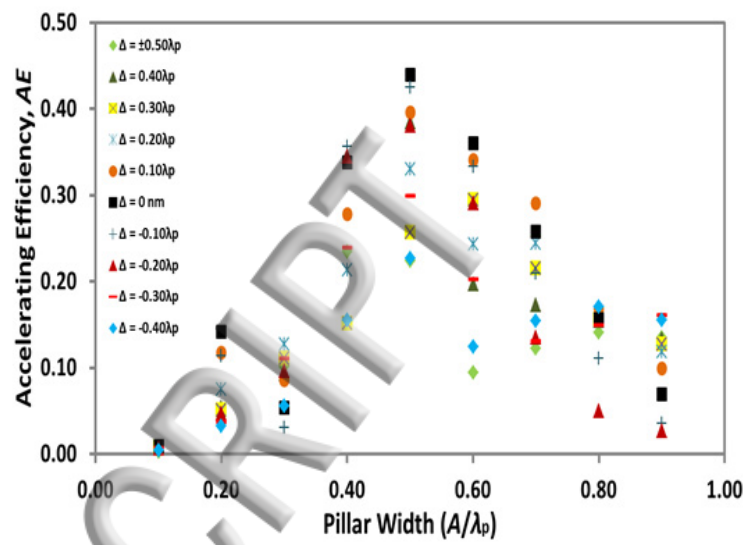

(b)

FIG. 2. Optimized accelerating factor $A F$ (a) and accelerating efficiency $A E$ (b) as a function of pillar width $A$ with variable longitudinal shift $\Delta$ for dual-gratings without a Bragg reflector: $C=0.50 \lambda_{\mathrm{p}}$ and $H=\lambda_{\mathrm{p}}$.

After optimization for a dual-grating structure, a Bragg reflector consisting of $N$ layers of dielectric material and $N-1$ layers of vacuum will be added into the simulation. The optical thicknesses in Fig. 3(b) are typically chosen to be a quarterwavelength long, that is, $n L_{1}=n_{0} L_{2}=\lambda_{0} / 4$ at laser wavelength $\lambda_{0}$, where $n$ and $n_{0}$ are the refractive index for dielectric and and vacuum layers in the Bragg reflector. Considering quartz $(n=1.5)$ as the dielectric material, power reflectance of $93 \%$, $99 \%$ and $99.7 \%$ are calculated for a Bragg reflector with $N=5,7$, and 9 respectively. Through adjusting the distance $D$ from 0 to $\lambda_{\mathrm{p}}$, the reflected field can constructively interfere with the subsequent input laser field. As illustrated in Fig. 3(c), the electric field along the vacuum channel center is apparently enhanced when adding a Bragg reflector to the dual-grating structure, thus boosting the accelerating gradient significantly. Figure 3(c) also shows that the longitudinal electric field for 7layer Bragg reflector is slightly higher than that of 5-layer reflector but keeps the same as that of 9-layer Bragg reflector. Thus a 7-layer Bragg reflector is chosen for our optimized structure. The maxima $A F=0.173$ and $A E=0.76$ are achieved at $C=0.50 \lambda_{\mathrm{p}}, H=\lambda_{\mathrm{p}}, A=0.50 \lambda_{\mathrm{p}}, \Delta=0 \mathrm{~nm}, D=0.80 \lambda_{\mathrm{p}}$. Considering that the damage threshold for quartz is $2 \mathrm{~J} / \mathrm{cm}^{2}$ for laser pulses of $100 \mathrm{fs}^{22,23}$, which is equivalent to an electric field of $E_{\mathrm{th}}=10 \mathrm{GV} / \mathrm{m}$, the maximum achievable gradient is $A F \times E_{\mathrm{th}}=$ $1.73 \mathrm{GV} / \mathrm{m}$ 


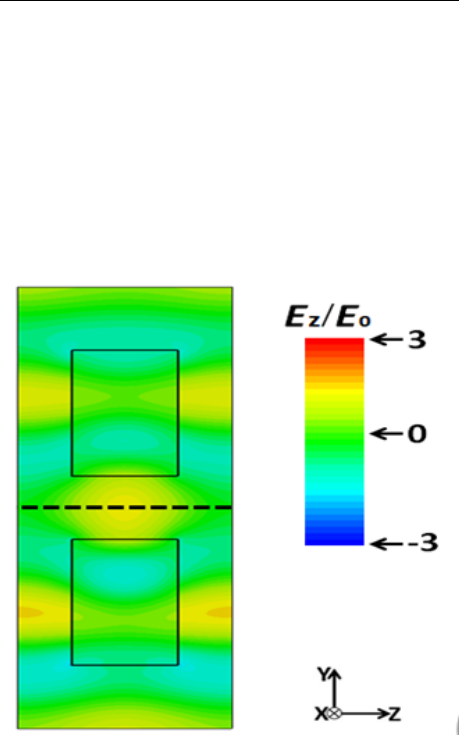

(a)

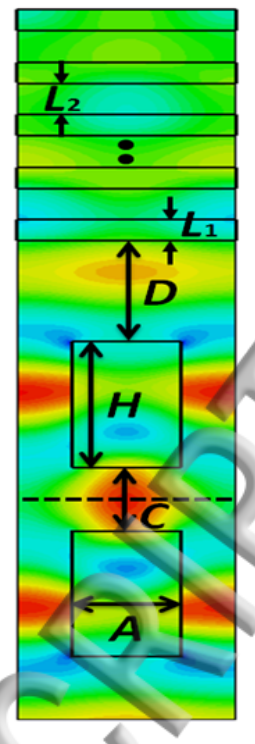

(b)

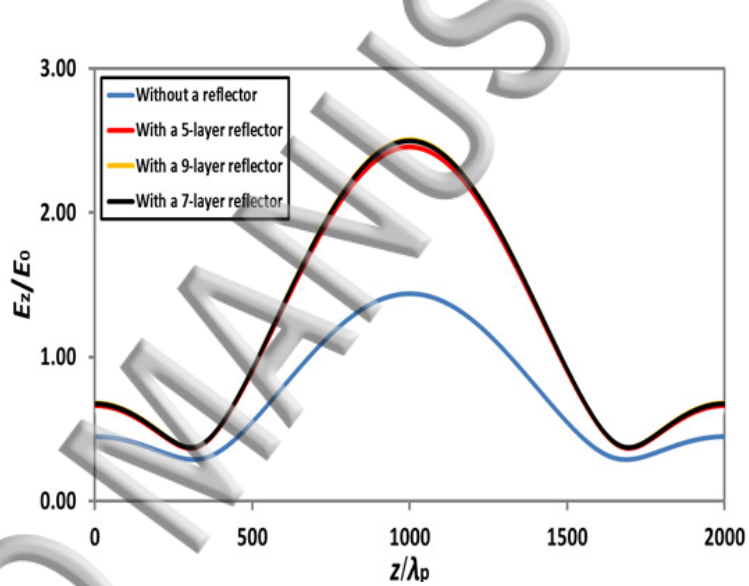

(c)

FIG. 3. Longitudinal electric field $E_{\mathrm{z}}$ distribution for a single-period structure, (a) without a Bragg reflector, (b) with a Bragg reflector, and (c) a comparison of both cases where a uniform laser field $E_{0}$ is used: $C=0.50 \lambda_{\mathrm{p}}, H=\lambda_{\mathrm{p}}, A=0.50 \lambda_{\mathrm{p}}, \Delta=0$ nm. Please note that the black line overlaps with the yellow line in the (c).

We also study the effect of rounded corners on the accelerating performance for such an optimized dual-grating structure with a Bragg reflector. In order to reduce the maximum field generated in the rectangular corners, rounded corners with a radius of $R$ are used as shown in the subplot of Fig. 4. It can be seen in Fig. 4 that $A F$ intially increases but then starts to decrease with larger radius, whereas $A E$ gradually decreases with increasing radius. The maximum $A F=0.185$ is achieved at a radius of $R=0.05 \lambda_{\mathrm{p}}$, while $A E$ peaks for rectangular pillars with $R=0.0 \mathrm{~nm}$. When a radius of $R=0.05 \lambda_{\mathrm{p}}$ is chosen for the rounded corners, we can get a maximum $A F=0.185$ which is an increase of 0.012 compared to the optimized rectangular dual-grating with a Bragg reflector, while $A E$ decreases from $A E=0.76$ to 0.75 . Considering that the accelerating factor limits the maximum achievable gradient, rounded corners with a radius of $R=0.05 \lambda_{\mathrm{p}}$ are chosen for our optimized structures. 


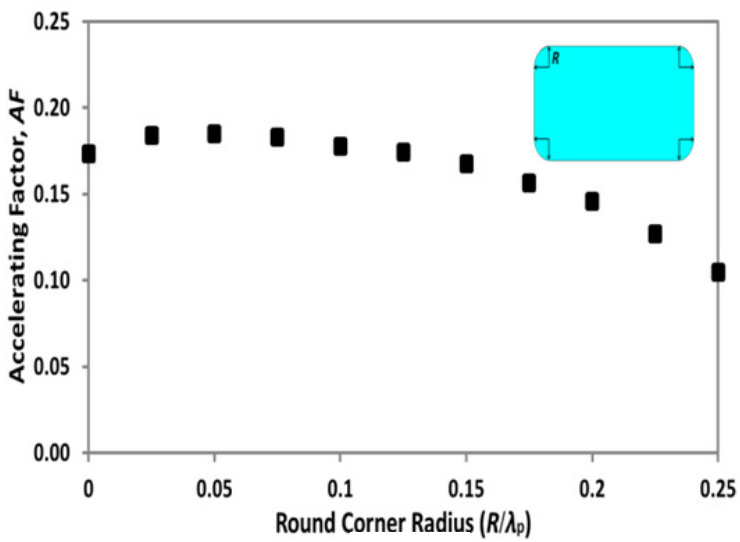

(a)

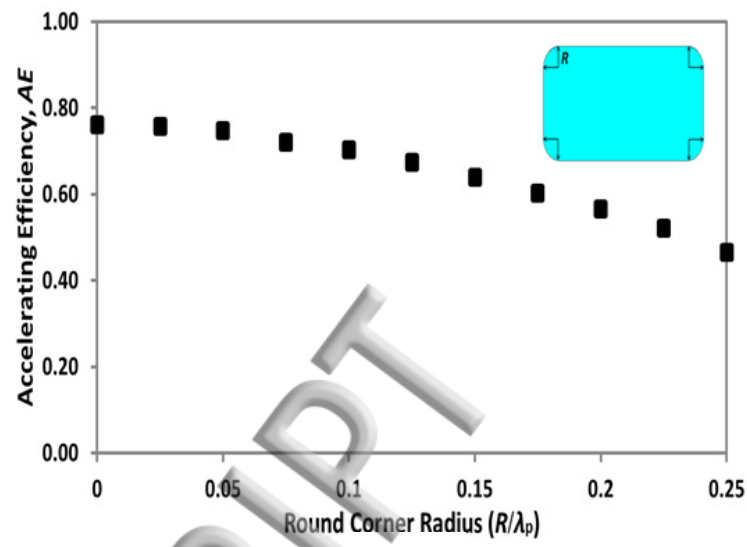

(b)

FIG. 4. Optimized accelerating factor $A F$ (a) and accelerating efficiency $A E$ (b) as a function of rounded corner radius $R$ for different structures.

Following the same procedure, structures with different vacuum channel gaps $C$ between $0.2 \lambda_{\mathrm{p}}$ and $\lambda_{\mathrm{p}}$ are optimized, with the aim of maximizing the accelerating factor or the accelerating efficiency. Figure 5(a) shows that dual-gratings with a 7layer Bragg reflector can generate a slightly larger accelerating factor than bare dual-gratings. The minor increments on the plot are in the range of $0.02 \sim 0.04$, which indicates that a Bragg reflector can increase the maximum achievable gradient by $0.18 \sim 0.36 \mathrm{GV} / \mathrm{m}$ for the $100 \mathrm{fs}-$ pulsed laser illumination. It can be seen in Fig. 5(b) that the accelerating efficiency is significantly improved by more than $70 \%$, when a Bragg reflector is added for dual-gratings. This means that dual-gratings with a Bragg reflector can be illuminated by a laser with $65 \%$ lower power to generate the same accelerating gradient compared to bare dual-grating structures. The maximum accelerating efficiency reaches as high as 0.91 , which is $80 \%$ higher than previously reported dual-grating structures which have a maximum accelerating efficiency of 0.50 for single laser beam illumination $^{1,2}$. For a channel gap $C=\lambda_{\mathrm{p}}$, the accelerating gradient can even be doubled when a Bragg reflector is used.

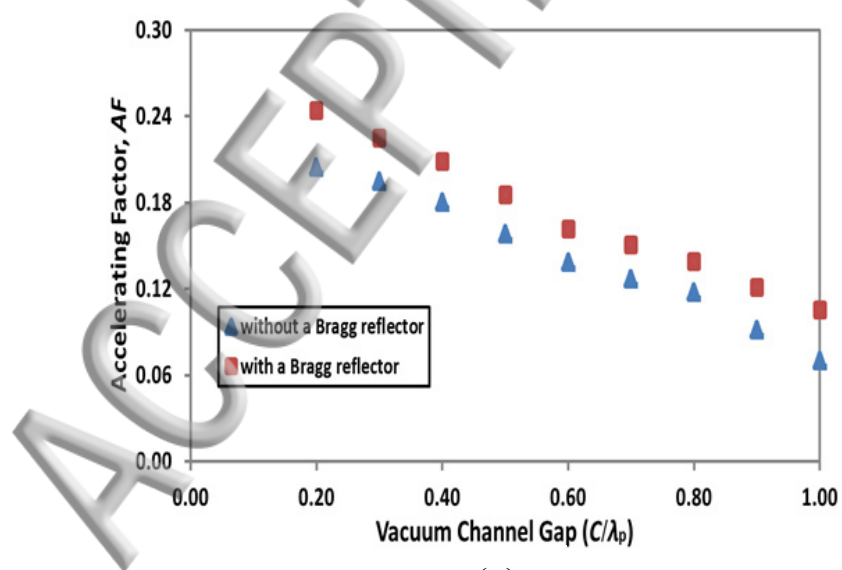

(a)

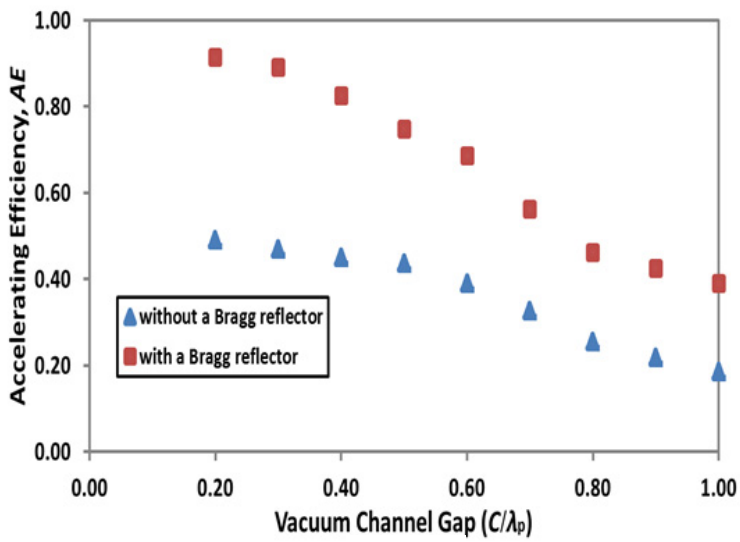

(b)

FIG. 5. Optimized accelerating factor $A F$ (a) and accelerating efficiency $A E$ (b) as a function of vacuum channel gap $C$ for different structures with rounded corners of $R=0.05 \lambda_{\mathrm{p}}$. 
AS a practical example, a dual-grating structure with a 7-layer Bragg reflector, with $C=0.50 \lambda_{\mathrm{p}}, H=\lambda_{\mathrm{p}}, A=0.50 \lambda_{\mathrm{p}}, \Delta=0$ $\mathrm{nm}, R=0.05 \lambda_{\mathrm{p}}, D=0.80 \lambda_{\mathrm{p}}, \lambda_{\mathrm{p}}=2.0 \mu \mathrm{m}$ is chosen as the optimum for our 2D particle-in-cell simulations. The electron bunch employed in our simulations has a mean energy of $50 \mathrm{MeV}$, bunch charge of $0.1 \mathrm{pC}$, RMS length of $9 \mu \mathrm{m}$, RMS radius of 10 $\mu \mathrm{m}$, normalised emittance of $0.2 \mathrm{~mm} \cdot \mathrm{mrad}$, and energy spread of $0.03 \%$. Such an electron bunch can be achieved from future Compact Linear Accelerator for Research and Applications (CLARA) ${ }^{24}$ or Advanced Superconducting Test Accelerator (ASTA) at Fermilab ${ }^{25}$. A uniform plane wave was used for our analytical studies in Section II; however, a Gaussian laser pulse, which can be represented as a superposition of plane waves with different $k$-vector magnitudes, is chosen for our 2D particle-in-cell simulations. Here, a laser pulse with wavelength $\lambda_{0}=2.0 \mu \mathrm{m}$, pulse energy $\Delta P=0.6 \mu \mathrm{J}$, pulse duration $\tau_{\mathrm{p}}=$ $100 \mathrm{fs}$, and waist radii $w_{\mathrm{x}}=10 \mu \mathrm{m}, w_{\mathrm{z}}=50 \mu \mathrm{m}$ would generate an input field $E_{0}=2 \mathrm{GV} / \mathrm{m}$. When such a laser field is used for illumination, the maximum electric field is still below the damage threshold for quartz structures. In a co-moving frame, the electrons experience a Gaussian-distributed temporal field $E_{\mathrm{t}}=G_{\mathrm{p}} e^{-\left(\frac{z}{w_{\text {int }}}\right)^{2}}$ with a characteristic interaction length $w_{\text {int }}=\left(\frac{1}{w_{\mathrm{z}}^{2}}+\frac{2 \ln 2}{\left(\beta c \tau_{\mathrm{p}}\right)^{2}}\right)^{-0.5}=22.7 \mu \mathrm{m}$, as described in Ref. 26. Considering $z=0$ to be the longitudinal center of a multiperiod dual-grating structure, we obtain an energy gain $\Delta E_{\mathrm{m}}=\int_{-0.5 L Z}^{0.5 L Z} q G_{\mathrm{P}} e^{-\left(\frac{z}{w_{\mathrm{int}}}\right)^{2}} d z$, where $L Z$ is the longitudinal length of a multi-period dual-grating structure and $q$ is the charge on a single electron. It is easily seen that for $L Z \geq 200 \mu \mathrm{m}$ a peak gradient of $G_{\mathrm{p}}=1.0 \mathrm{GV} / \mathrm{m}$ results in a energy gain of $\Delta E_{\mathrm{m}} \approx \sqrt{\pi} w_{\mathrm{int}}=40 \mathrm{keV}$, which is used to calculate the loaded gradient for subsequent analysis. In order to save computing time, a 100-period optimum structure with a length $L Z=200 \mu \mathrm{m}$ is chosen for our particle-in-cell simulations.
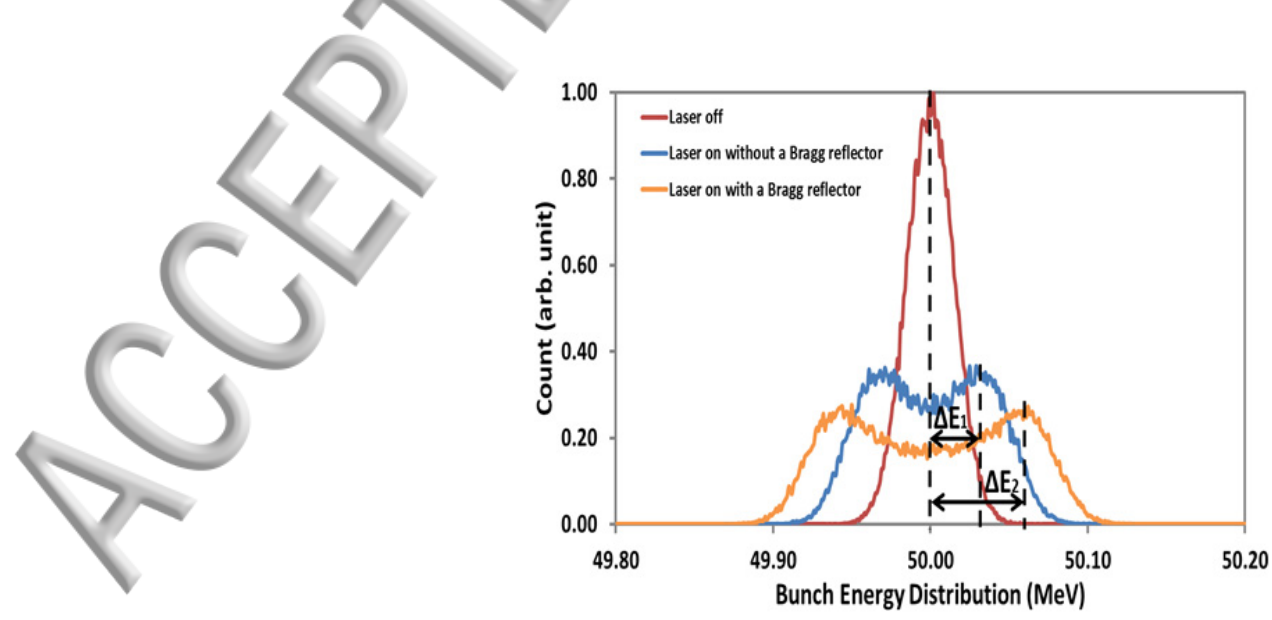

FIG. 6. Bunch energy distribution for three cases: laser-off (red line); laser-on without a Bragg reflector (blue line); laser-on with a Bragg reflector (orange line). 

used for our calculations. It is found that about $4 \%$ of the $50 \mathrm{MeV}$ bunch is transmitted through the vacuum channel gap of 1.0 $\mu \mathrm{m}$. Figure 6 shows the bunch energy distribution for those modulated electrons with the laser off and on. It can be seen that the energy sprectrum has a double-peaked profile after laser-bunch interaction due to longer bunch RMS length of $9 \mu \mathrm{m}$ than laser wavelength, which agrees well with the reported results ${ }^{11,12,28}$. The maximum energy gain is $\Delta E_{2}=59 \pm 4 \mathrm{keV}$ for optimized dual-gratings with a Bragg reflector, while it is $\Delta E_{1}=32 \pm 4 \mathrm{keV}$ for bare dual-gratings. This corresponds to loaded peak gradients of $1.48 \pm 0.10 \mathrm{GV} / \mathrm{m}$ and $0.80 \pm 0.10 \mathrm{GV} / \mathrm{m}$, respectively. The loaded gradient is therefore increased by $(85 \pm 26) \%$ when a Bragg reflector is added, for optimized dual-gratings.

\section{Diffraction Effect}

Our 2D analytical optimizations in Section II and particle-in-cell simulations in Section III are based on the assumption that the electromagnetic field is invariant along the $x$-direction and hence that the field has a 2D solution. In reality, the fabricated vertical size $J$ as shown in Fig. 7 plays an important role in the accelerating performance for such an optimized structure. For an incident laser with a transverse waist radius $w_{\mathrm{x}}=10 \mu \mathrm{m}$, the fabricated vertical size $J$ should ideally be more than $20 \mu \mathrm{m}$. It would however be quite challenging to fabricate a Bragg reflector with such a high aspect ratio as $J / L_{1}=$ 60 based on existing quartz etching technology, although etching to a depth of over $100 \mu \mathrm{m}$ with vertical sidewalls has been demonstrated in Ref. 29-31. In this case, diffraction effects may alter the distribution of a reflected laser beam when the fabricated vertical size $J$ is smaller than the transverse laser waist radius $w_{\mathrm{x}}$. As illustrated in Fig. 7, the longitudinal electric field in the channel center is not evenly distributed along the $x$-direction, thereby reducing the accelerating performance when electrons are travelling along the channel center. 


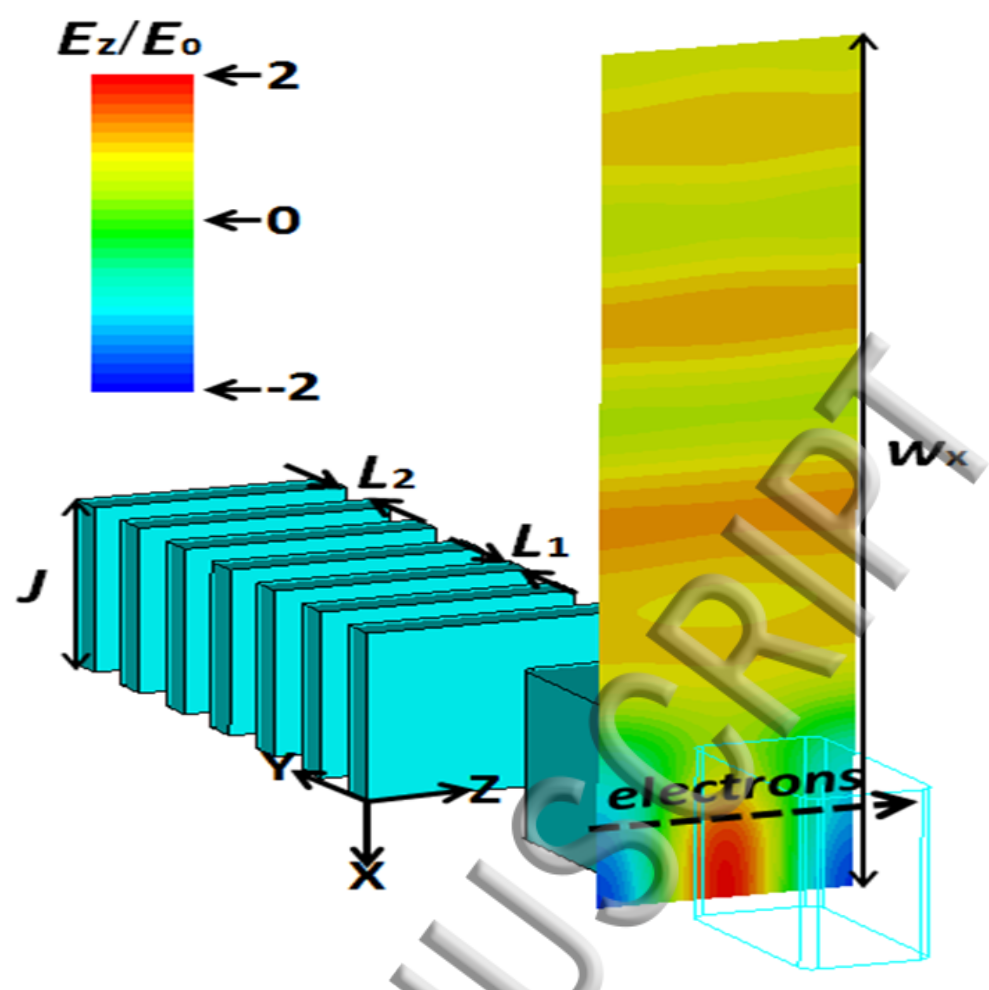

FIG. 7. Transverse profile of the longitudinal electric field at the channel center for the optimized structures of a vertical size $J / w_{\mathrm{x}}=0.2$. The green wireframe is the outline of the other grating, which is hidden in order to show the field distribution clearly.

The relationship between the fabricated vertical size $J / w_{\mathrm{x}}$, the accelerating factor $A F$, and accelerating efficiency $A E$ are then studied, which are shown in Fig. 8. It is found that the maximum $A F=0.185$ can be achieved at $J / w_{\mathrm{x}}=1.0$, while $A E$ peaks at $J / w_{\mathrm{x}}=0.4$. When $J / w_{\mathrm{x}}$ equals 0.10 , we get $A F=0.08$ and $A E=0.47$, which are comparable with the values for simulated 2D dual-gratings without a Bragg reflector. In this situation a Bragg reflector does not work at all. However, when $J / w_{\mathrm{x}}$ equals 0.20 , the Bragg reflector starts to work and we can get $A F=0.11$ and $A E=0.67$. The fabricated vertical size $J$ should therefore be at least $0.20 w_{\mathrm{x}}$ if an acceptable accelerating performance is to be expected. For example, for an incident laser waist radius of $w_{\mathrm{x}}=10 \mu \mathrm{m}$, an optimized structure can be fabricated with a vertical size $J \geq 2 \mu \mathrm{m}$ so that we can get an aspect ratio of $J / L_{1} \geq 6$ for such a Bragg reflector. Considering that deep etching into quartz of over $100 \mu \mathrm{m}$ with an aspect ratio of over 10 to 1 has been demonstrated in Ref. 31, a Bragg reflector with an aspect ratio of $J / L_{1} \geq 6$ can be achieved using existing quartz etching technology. For a vertical size $J=2 \mu \mathrm{m}$, the analytically-calculated gradient is $0.67 E_{0}$, which is a reduction of $11 \%$ from the value of $0.75 E_{0}$ for $2 \mathrm{D}$ optimized structures. The resulting loaded gradient can in principle be decreased from $1.48 \pm 0.10$ to $1.32 \pm 0.09 \mathrm{GV} / \mathrm{m}$, based on the same particle-in-cell simulation as in Section III. However, due to the small accelerating factor $A F=0.11$, the maximum achievable gradient is limited to $1.10 \mathrm{GV} / \mathrm{m}$. This corresponds to an incident laser with an input field of $1.64 \mathrm{GV} / \mathrm{m}$, which generates a maximum field close to the damage threshold in the quartz structure. 


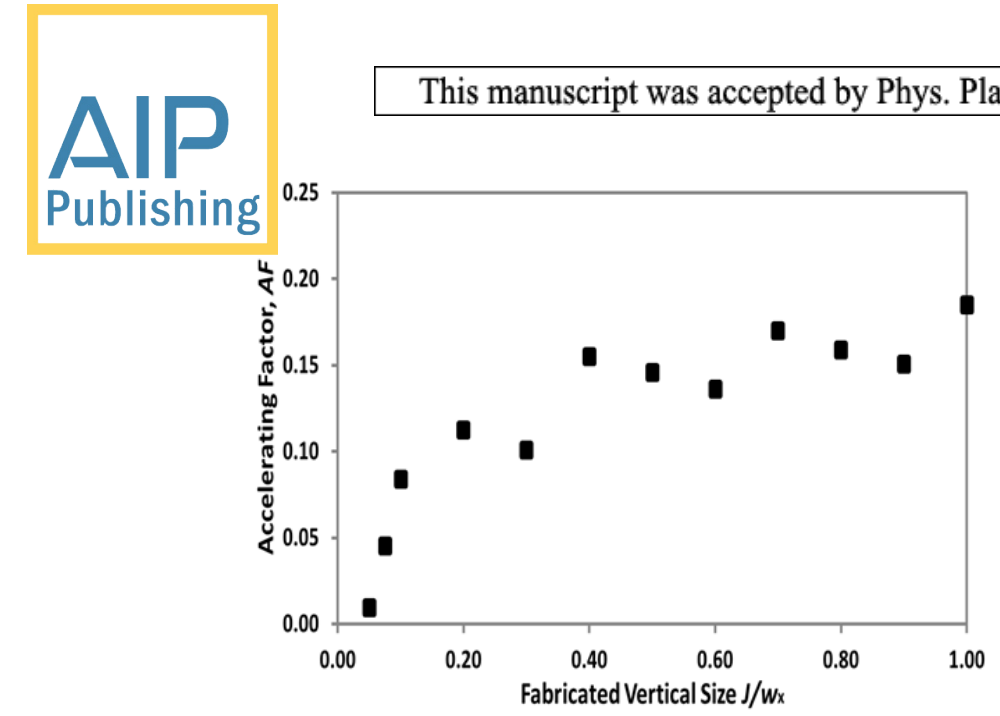

(a)

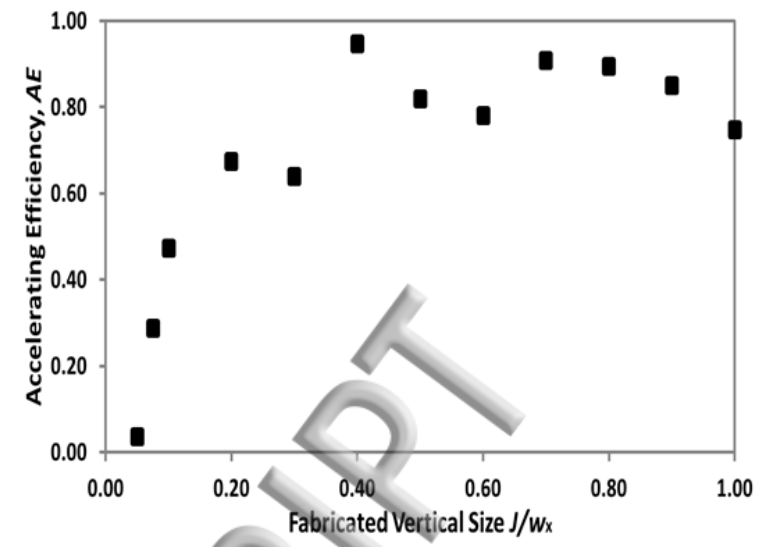

(b)

FIG. 8. Optimized accelerating factor $A F$ (a) and accelerating efficiency $A E$ (b) as a function of fabricated vertical size of $J$ which is normalized to the transverse laser waist radius $w_{\mathrm{X}}$.

\section{Conclusion}

In conclusion, we have studied numerically an efficient DLA structure based on dual-gratings with a Bragg reflector. The analytical results show that this structure can generate a $70 \%$ higher accelerating gradient for the same input laser fluence, and an accelerating factor slightly larger by $0.02 \sim 0.04$, than bare dual-gratings. Despite the small improvement in accelerating factor, this design could yield the same accelerating gradient when operated at a $65 \%$ lower laser power, compared to that reported for dual-gratings. Moreover, 2D particle-in-cell simulations have been run in which a $50 \mathrm{MeV}$ electron bunch is loaded into an optimized 100-period structure to interact with a laser pulse. A loaded accelerating gradient of $1.48 \pm 0.10 \mathrm{GV} / \mathrm{m}$ can be achieved, which is $(85 \pm 26) \%$ higher than that of bare dual-gratings for an input laser field of 2 $\mathrm{GV} / \mathrm{m}$. Finally, studies of the diffraction effect due to a fabricated vertical size $J$ smaller than the laser waist radius have also been presented. It is found that the optimized structure should be fabricated with a vertical size $J / w_{\mathrm{x}} \geq 0.20$, to generate an acceptable accelerating performance.

By extension, a Bragg reflector can be added to improve the accelerating efficiency for any DLA structure. In addition, such a Bragg reflector can be integrated with any DLA structure into a single wafer, using existing nanofabrication technology. This eliminates the complicated alignment process involved when a mirror is used externally to reflect the laser power back into any structure. However, realistic fabrication studies are still required to pave the way for implementing the proposed concept for such an integrated nano-structure.

\section{Acknowledgements}

We would like to thank Dr. Mark Ibison for carefully proof reading the original manuscript. This work was supported by the EU under Grant Agreement 289191 and STFC Cockcroft Institute core grant No.ST/G008248/1. 
${ }^{3}$ A. Aimidula, C. P. Welsch, G. Xia, K. Koyama, M. Uesaka, M. Yoshida, O. Mete, Y. Matsumura, Nucl. Instrum. Methods Phys. Res., Sect. A, 740, 108 (2014).

${ }^{4}$ C. M. Chang and O. Solgaard, Appl. Phys. Lett. 104, 184102 (2014).

${ }^{5}$ X. E. Lin, Phys. Rev. ST Accel. Beams 4, 051301 (2001).

${ }^{6}$ V. Reboud, J. Romero-Vivas, P. Lovera, N. Kehagias, T. Kehoe, G. Redmond, and C. M. S. Torres, Appl. Phys. Lett. 102, 073101 (2013).

${ }^{7}$ B. M. Cowan, Phys. Rev. ST Accel. Beams 6, 101301 (2003).

${ }^{8}$ S. Y. Lin, J. G. Fleming, D. L. Hetherington, B. K. Smith, R. Biswas, K. M. Ho, M. M. Sigalas, W. Zubrzycki, S. R. Kurtz \& Jim Bur, Nature 394, 251 (1998).

${ }^{9}$ B. M. Cowan, Phys. Rev. ST Accel. Beams 11, 011301 (2008).

${ }^{10}$ Z. Wu, R.J. England, C.K. Ng, B. Cowan, C.McGuinness, C.Lee, M.Qi, and S. Tantawi, Phys. Rev. ST Accel. Beams 17, 081301 (2014).

${ }^{11}$ E. A. Peralta, K. Soong, R. J. England, E. R. Colby, Z. Wu, B. Montazeri, C. McGuinness, J. McNeur, K. J. Leedle, D. Walz, E. B. Sozer, B. Cowan, B. Schwartz, G. Travish and R. L. Byer, Nature 503,91(2013).

${ }^{12}$ K. P. Wootton, Z. Wu, B. M. Cowan, A. Hanuka, I. V. Makasyuk, E. A. Peralta, K. Soong, R. L. Byer, and R. J. England, Opt. Lett., 41, 2696 (2016).

${ }^{13}$ J. Breuer and P. Hommelhoff, Phys. Rev. Lett. 111, 134803 (2013).

${ }^{14}$ K. J. Leedle, R. F. Pease, R. L. Byer, and J. S. Harris, Optica 2, 158 (2015).

${ }^{15}$ K. J. Leedle, A. Ceballos, H. Deng, O. Solgaard, R. F. Pease, R. L. Byer, and J. S. Harris, Opt. Lett. 40, 4344 (2015).

${ }^{16}$ T. Plettner, R. L. Byer, and B. Montazeri, J. Mod. Opt. 58, 1518 (2011).

${ }^{17}$ E. Prat, S. Bettoni, M. Calvi, M. Dehler, F. Frei, P. Hommelhoff, M. Kozak, J. McNeur, C.Ozkan Loch, S. Reiche, A. Romann, R. Ischebeck, Nucl. Instrum. Methods Phys. Res., Sect. A (2017, in press); available at https://doi.org/10.1016/j.nima.2017.01.016.

${ }^{18}$ B. Cui, Z. Yu, H. Ge, and S. Chou, Appl. Phys. Lett. 90, 043118 (2007).

${ }^{19}$ R. Ghodssi and P. Lin, MEMS Materials and Processes Handbook (Springer, Berlin, 2011).

${ }^{20}$ VSim, available from https://www.txcorp.com/vsim.

${ }^{21}$ R. J. England, R. J. Noble, K. Bane, D. H. Dowell, C.-K. Ng, J. E. Spencer, S. Tantawi, Z. Wu, R. L. Byer, E. Peralta, K. Soong, C. Chang, B. Montazeri, S. J. Wolf, B. Cowan, J. Dawson, W. Gai, P. Hommelhoff, Y. Huang, C. Jing, C. McGuinness, R. B. Palmer, B. Naranjo, J.Rosenzweig, G. Travish, A. Mizrahi, L. Schachter, C. Sears, G. R. Werner, and R. B. Yoder, Rev. Mod. Phys. 86, 1337 (2014).

${ }^{22}$ M. Lenzner, J. Krüger, S. Sartania, Z. Cheng, Ch. Spielmann, G. Mourou, W. Kautek, F. Krausz, Phys. Rev. Lett. 80, 18 (1998).

${ }^{23}$ K. Soong, R. L. Byer, C. McGuinness, E. Peralta, and E. Colby, in Proceedings of 2011 Particle Accelerator Conference (PAC2011) (IEEE, New York, USA, 2011), MOP095.

${ }^{24}$ J. A. Clarke, D. Angal-Kalinin, N. Bliss, R. Buckley, S. Buckley, R. Cash, P. Corlett, L. Cowie, G. Cox, G. P. Diakun, D. J. Dunning, B. D. Fell, A. Gallagher, P. Goudket, A. R. Goulden, D. M. P. Holland, S. P. Jamison, J. K. Jones, A. S. Kalinin, W. Liggins, L. Ma, K. B. Marinov, B. Martlew, P. A. McIntosh, J. W. McKenzie, K. J. Middleman, B. L. Militsyn, A. J. Moss, B. D. Muratori, M. D. Roper, R. Santer, Y. Saveliev, E. Snedden, R. J. Smith, S. L. Smith, M. Surman, T. Thakker, N. R. Thompson, R. Valizadeh, A. E. Wheelhouse, P. H. Williams, R. Bartolini, I. Martin, R. Barlow, A. Kolano, G. Burt, S. Chattopadhyay, D. Newton, A. Wolski, R. B. Appleby, H. L. Owen, M. Serluca, G. Xia, S. Boogert, A. Lyapin, L. Campbell, B. W. J. McNeil and V. V. Paramonov, J. Instrum. 9, T05001 (2014).

${ }^{25}$ P. Piot, V. Shiltsev, S. Nagaitsev, M. Church, P. Garbincius, S. Henderson, J. Leibfritz, arXiv:1304.0311 (2013).

${ }^{26}$ J. Breuer, R. Graf, A. Apolonski, and P. Hommelhoff, Phys. Rev. ST Accel. Beams 17, 021301 (2014).

${ }^{27}$ C. Warner III and F. Rohrlich, Phys. Rev. 93, 406(1954).

${ }^{28}$ Y. Wei, S. Jamison, G. Xia, K. Hanahoe, Y. Li, J. D. A. Smith, and C. P. Welsch, Phys. Plasmas 24, 023102 (2017).

${ }^{29}$ Y. Morikawa, T. Koidesawa, T. Hayashi, K. Suu, Thin Solid Films 515, 4918 (2007).

${ }^{30}$ Y. Tang, Y. Lin, T. Huang, J. Wang, M. Shiao and C. Yu, in 10th IEEE International Conference on Nano/Micro Engineered and Molecular Systems, Xi'an, 2015, pp. 401-404 (2015). DOI: 10.1109/NEMS.2015.7147453.

${ }^{31}$ M. Pedersen, M. Huff, Journal of Microelectromechanical Systems 26, 448 (2017). 


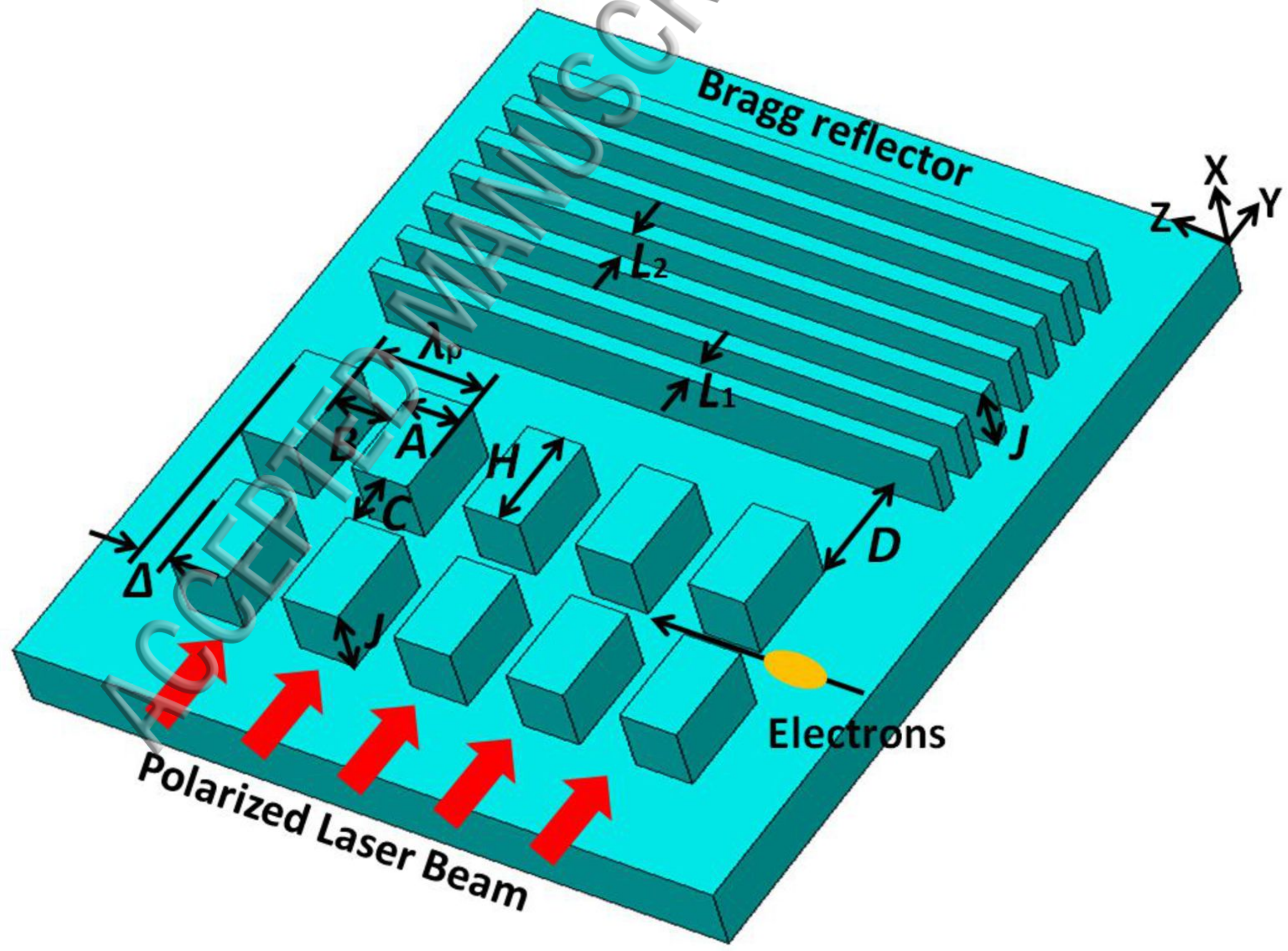




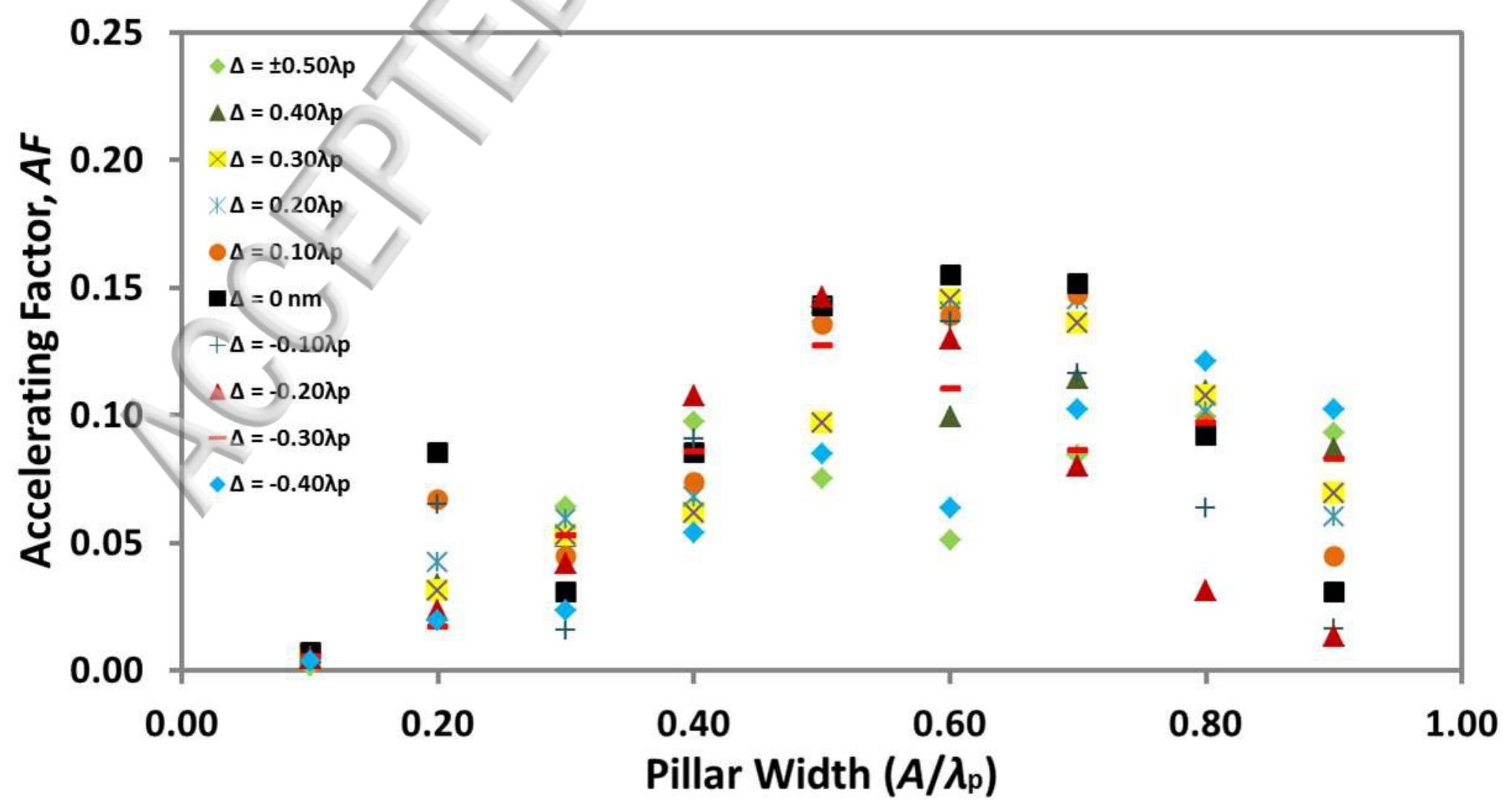




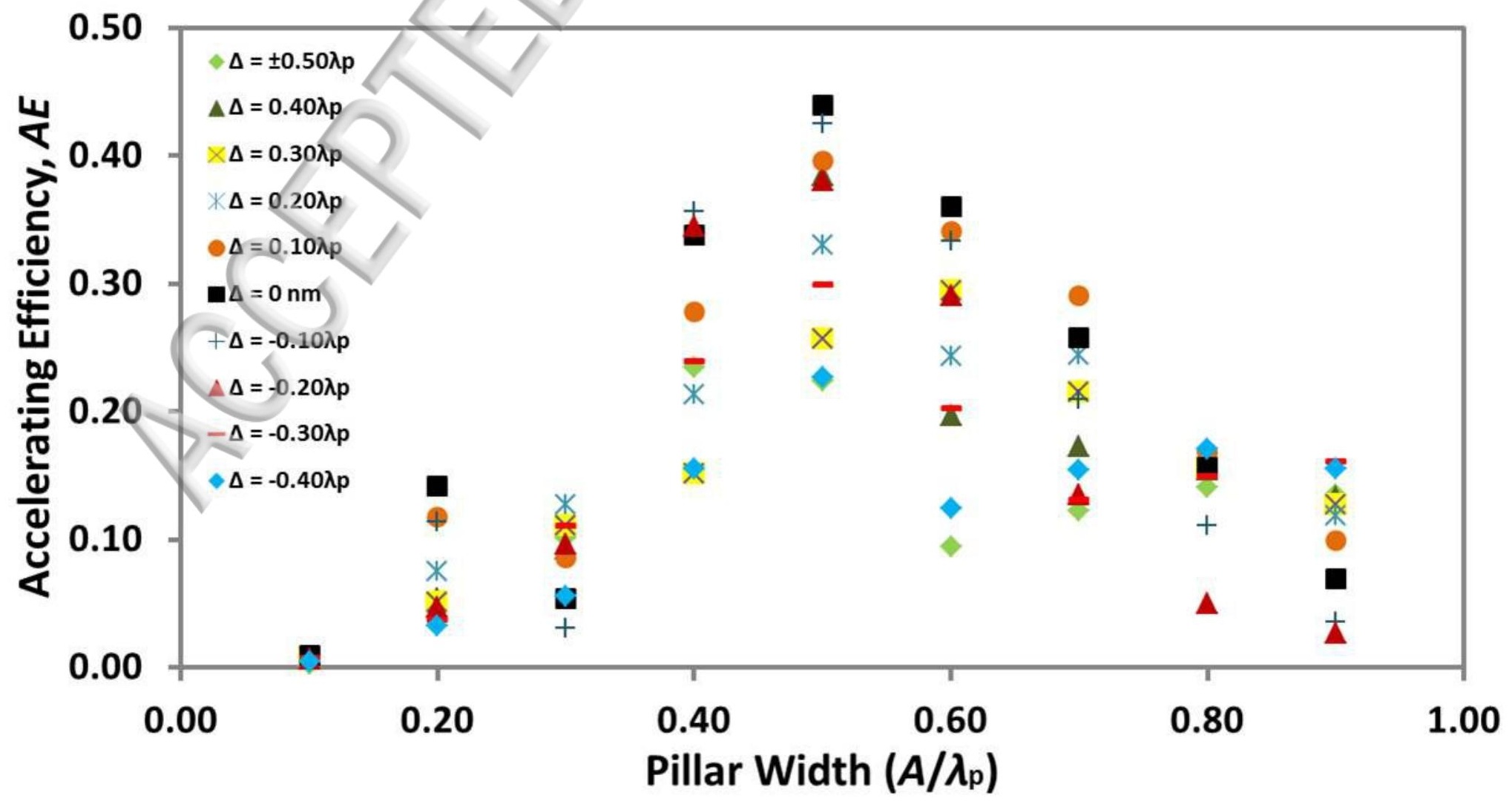




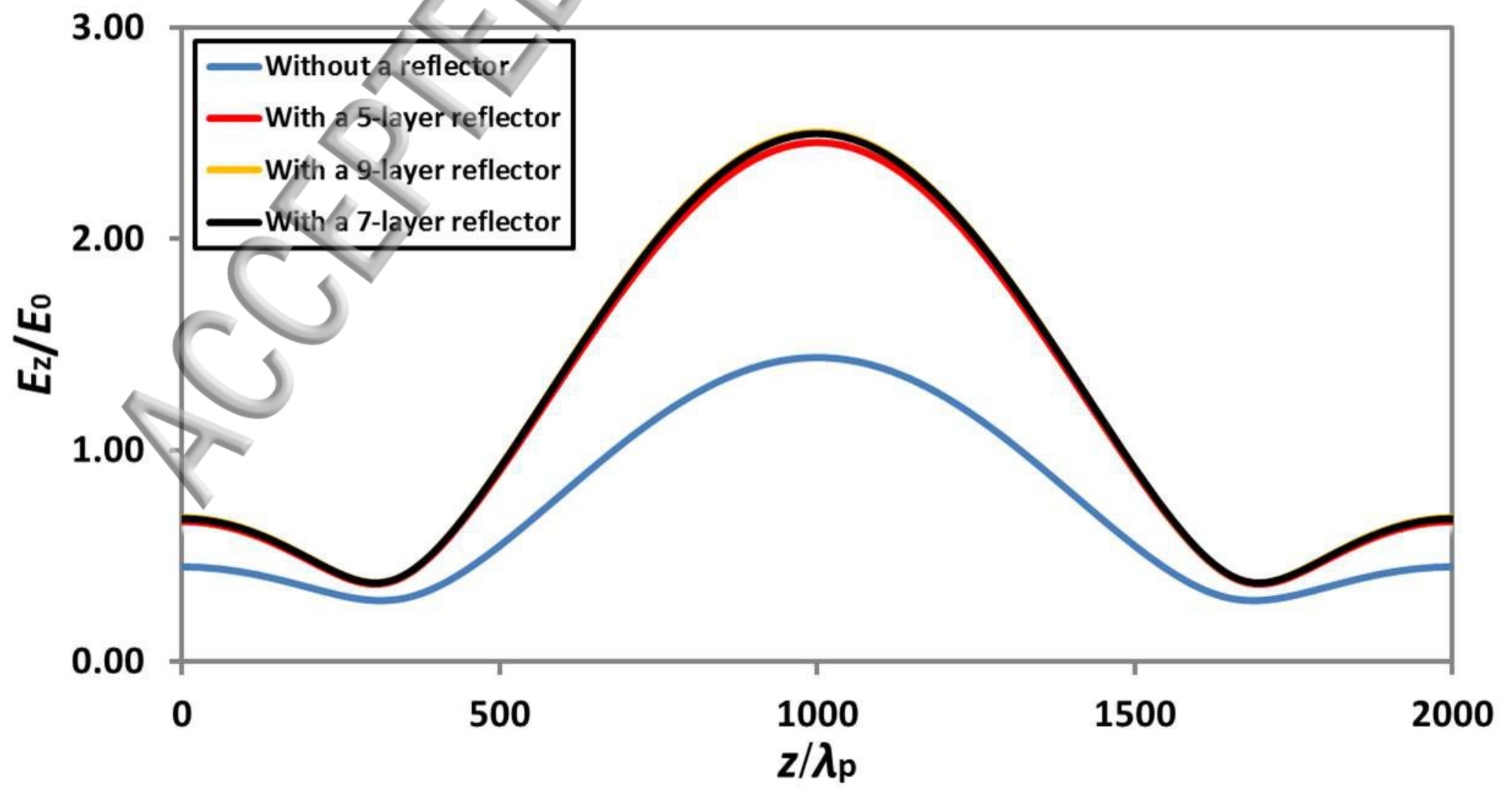




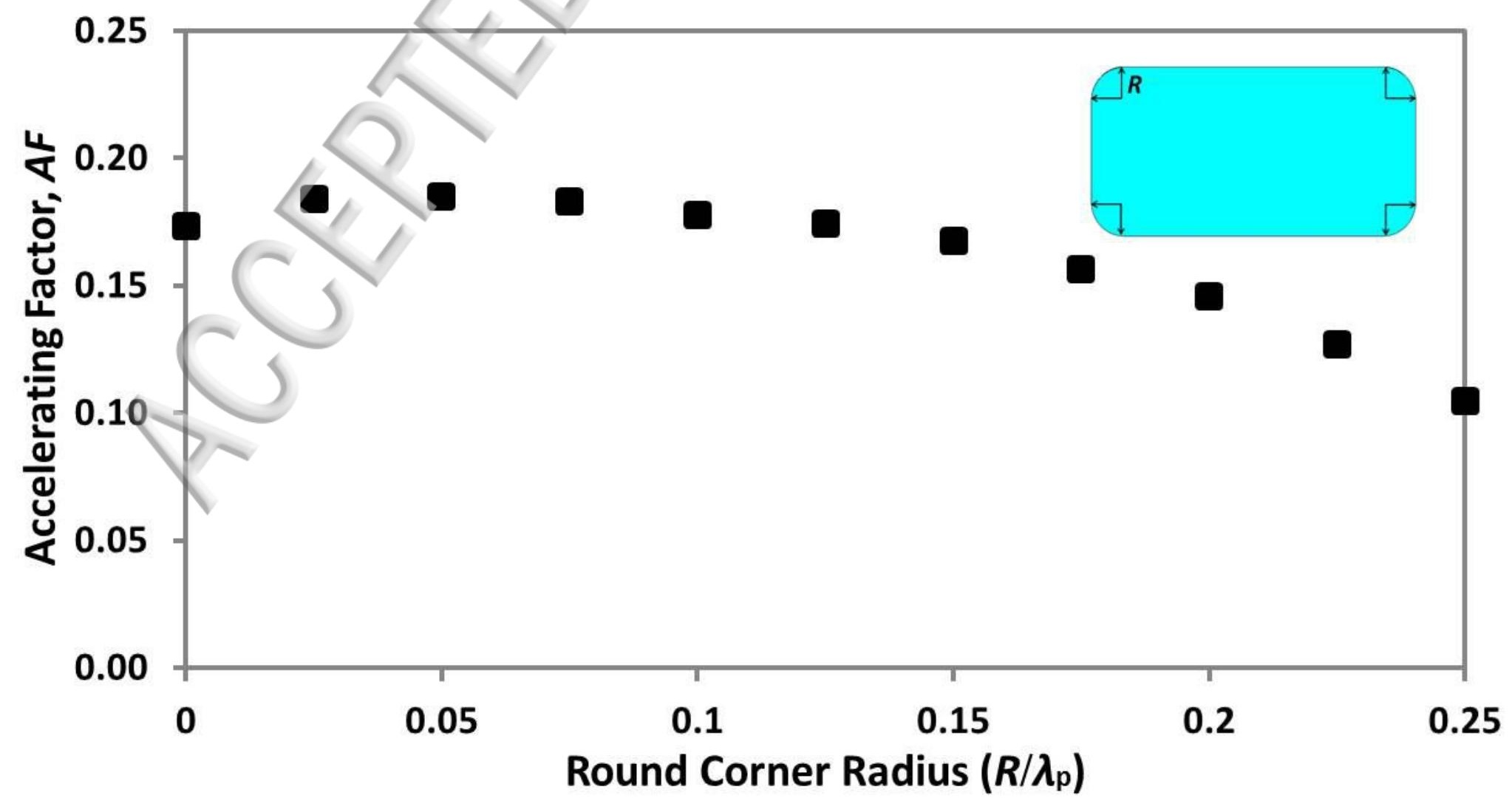




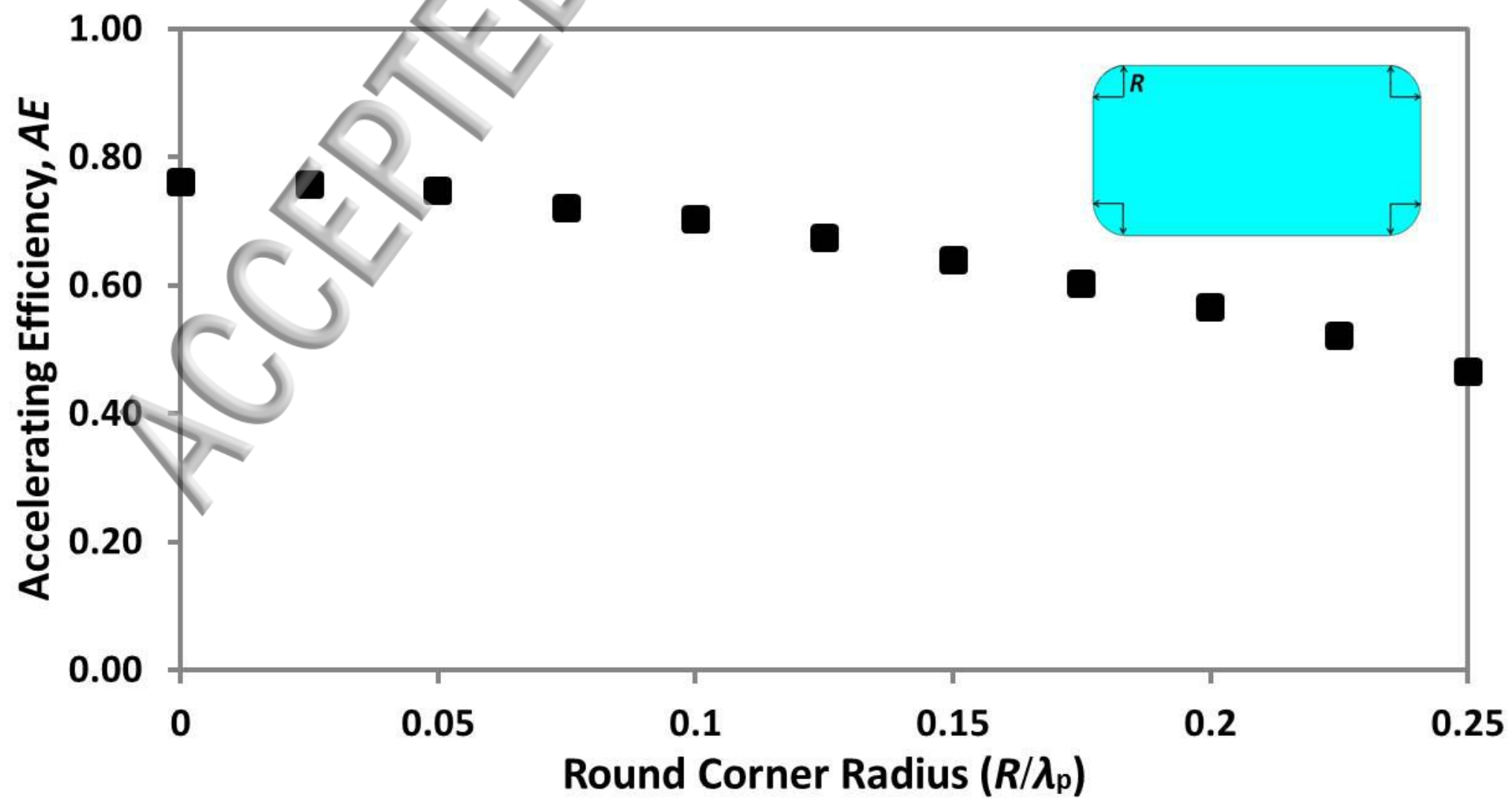




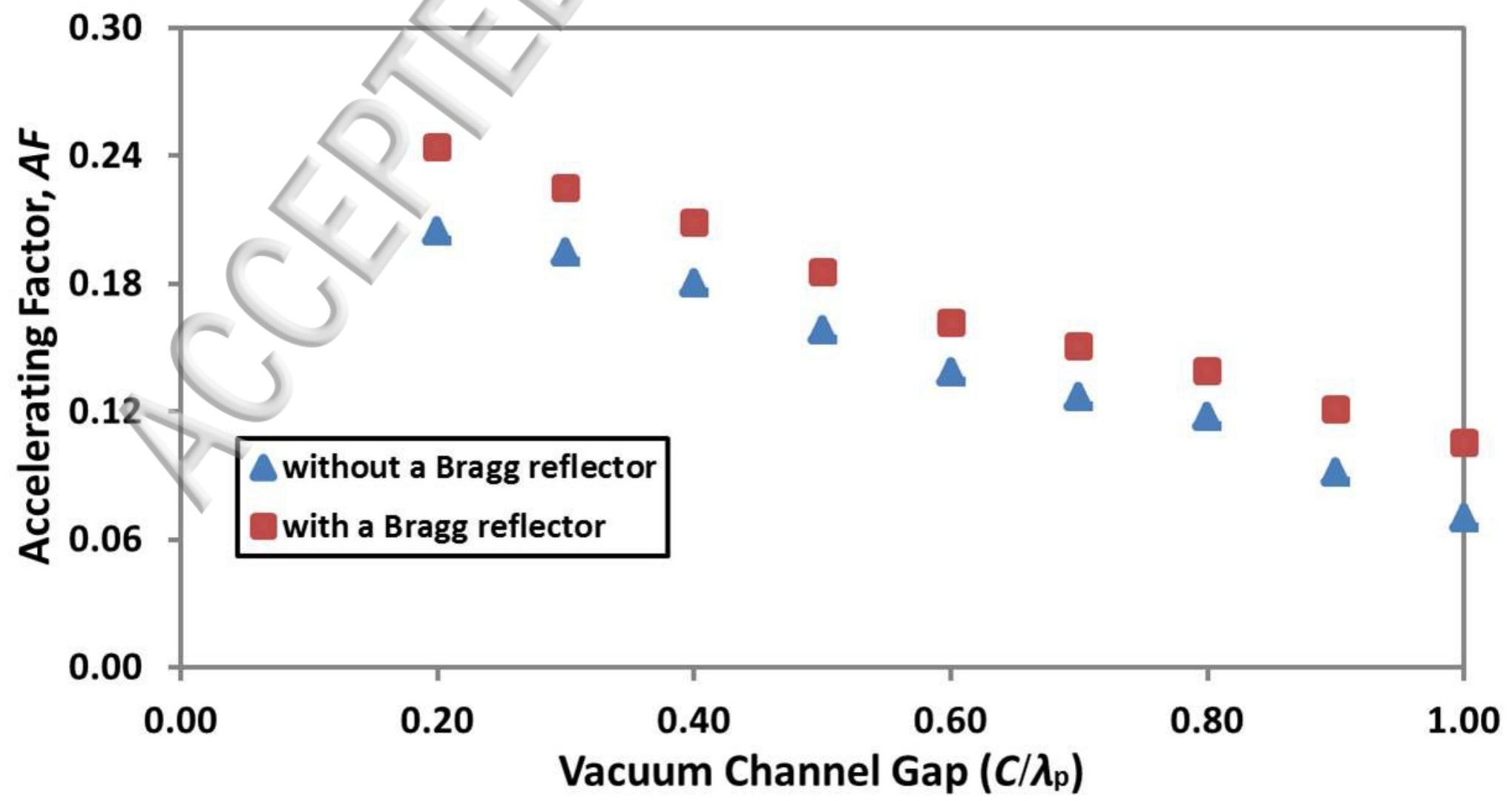




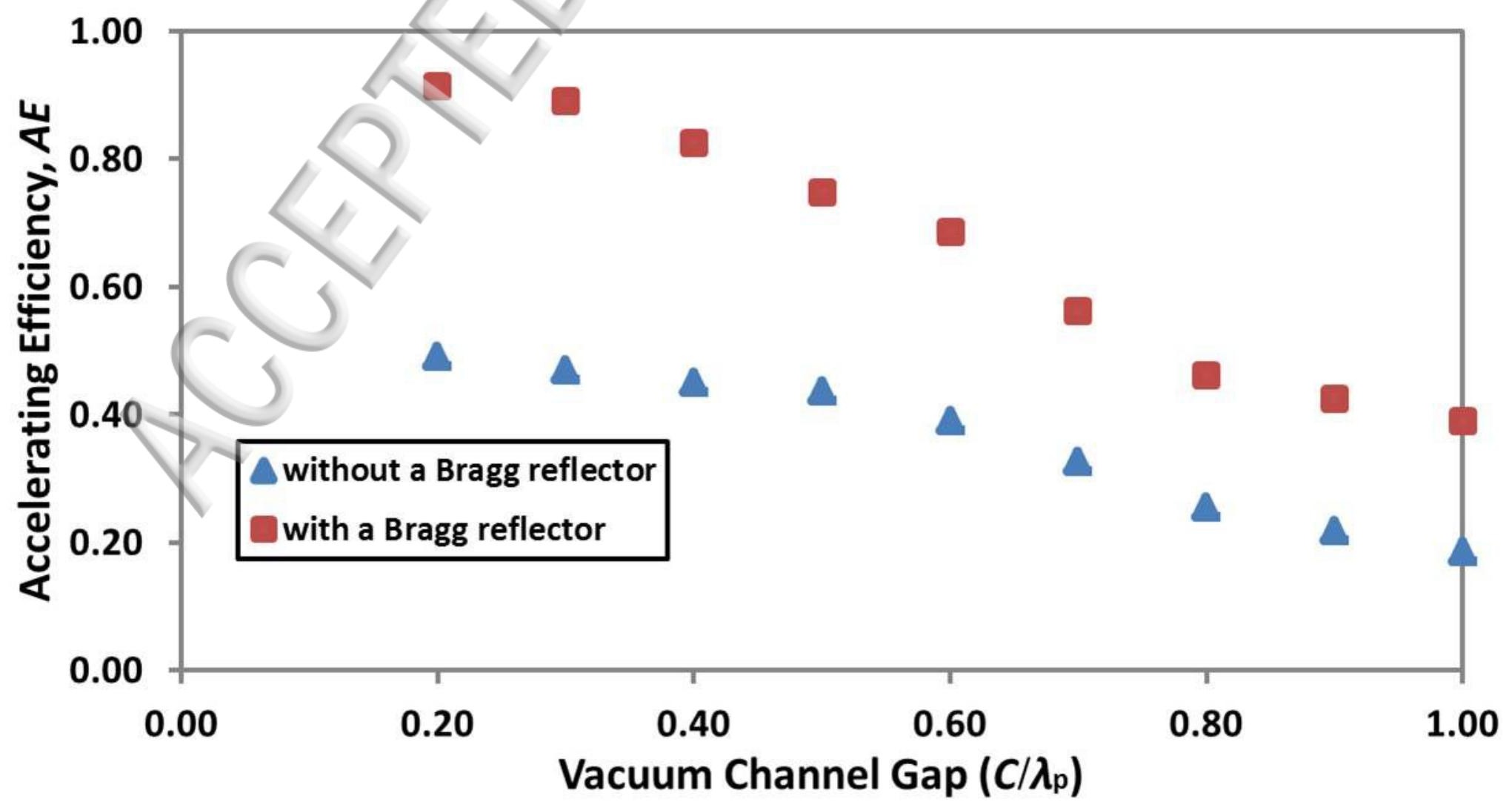




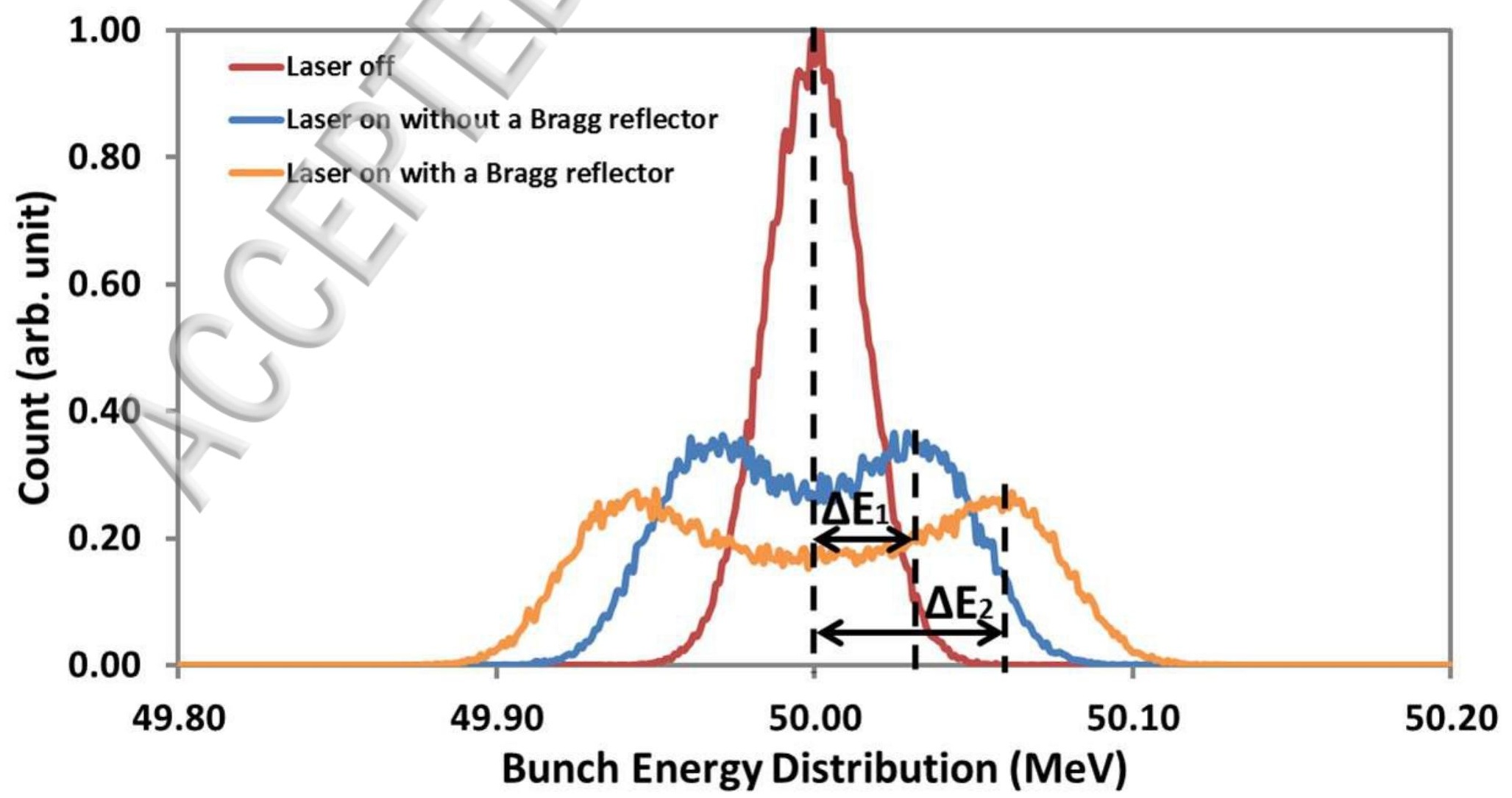




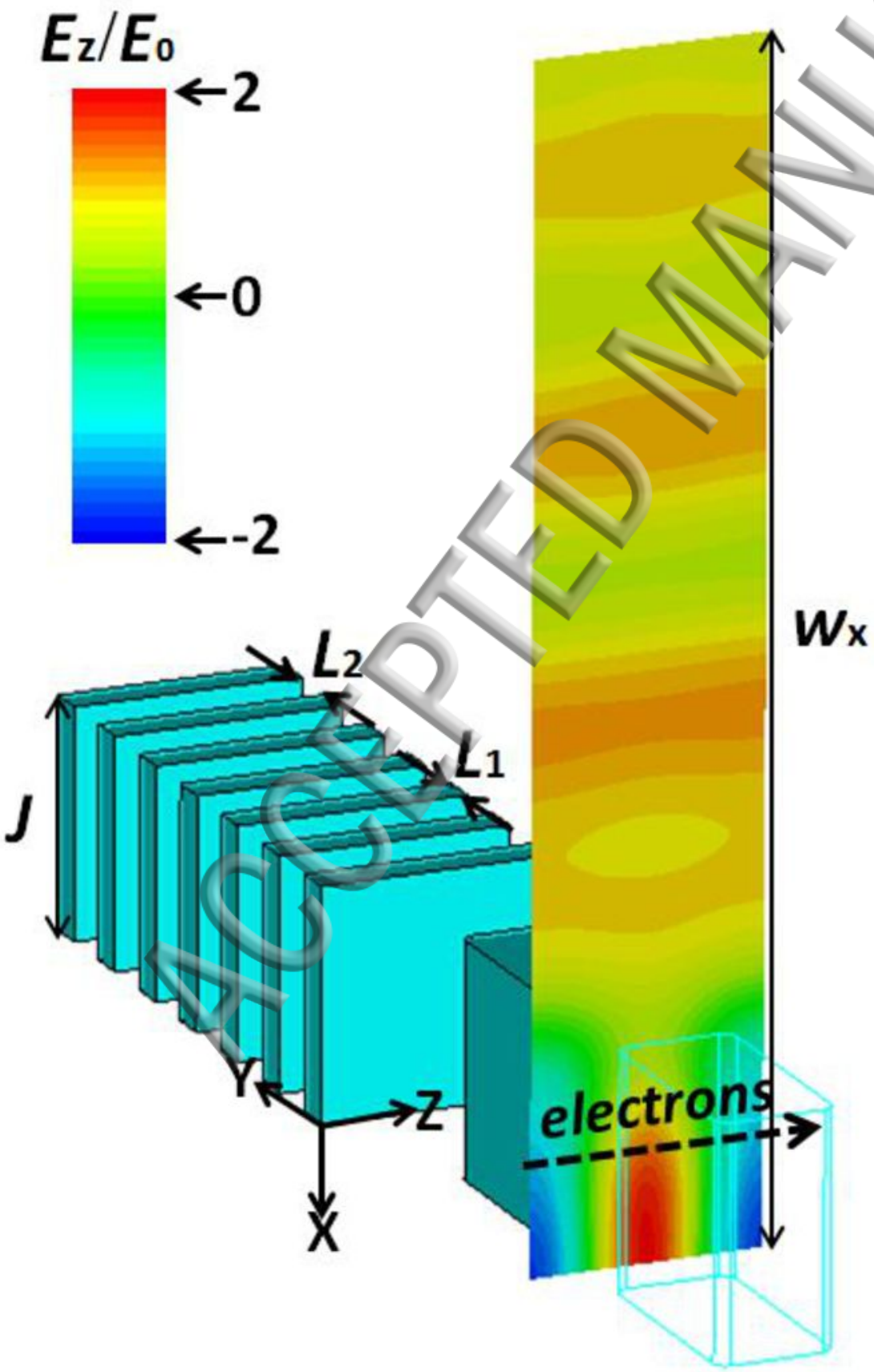




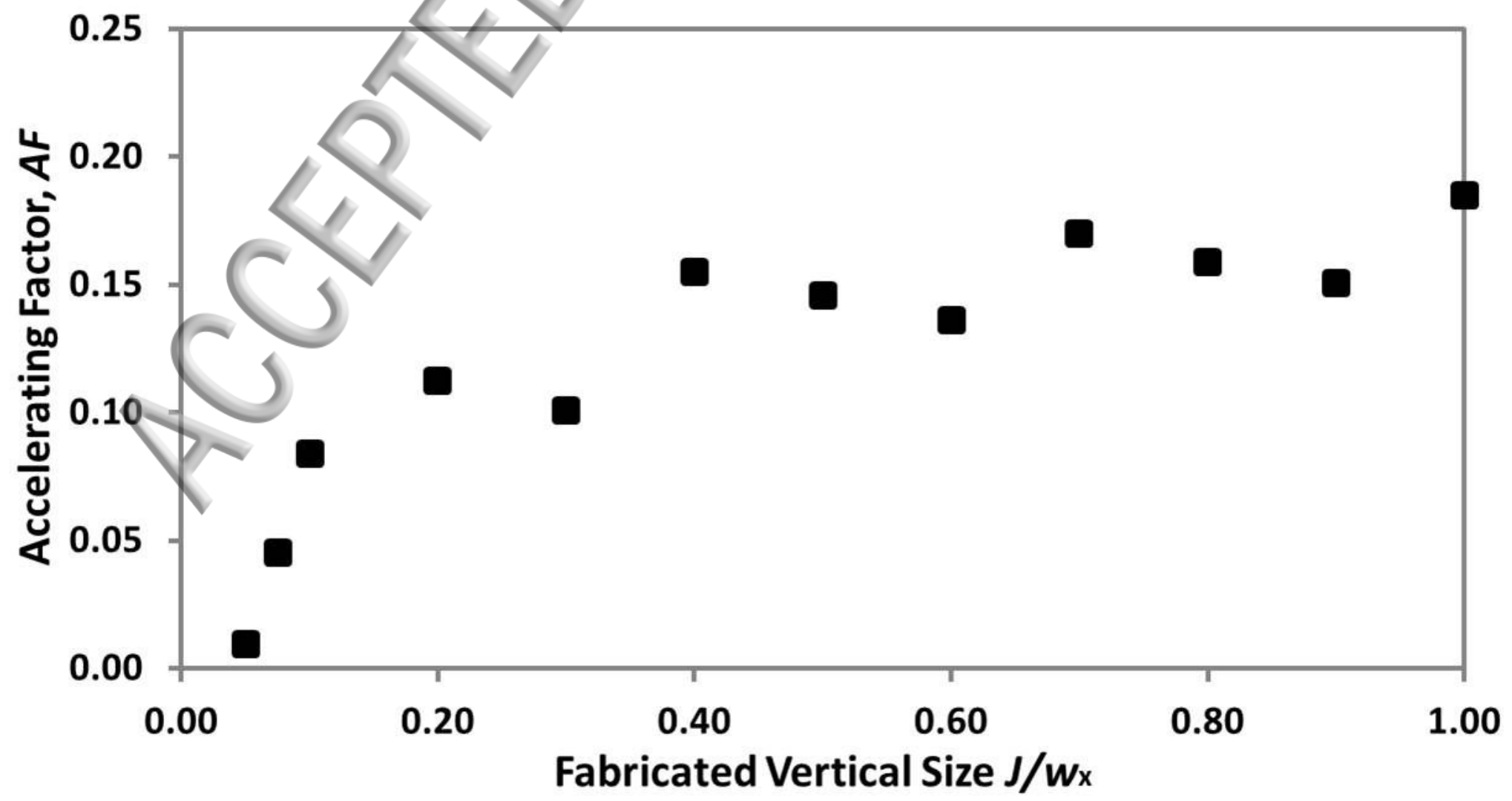




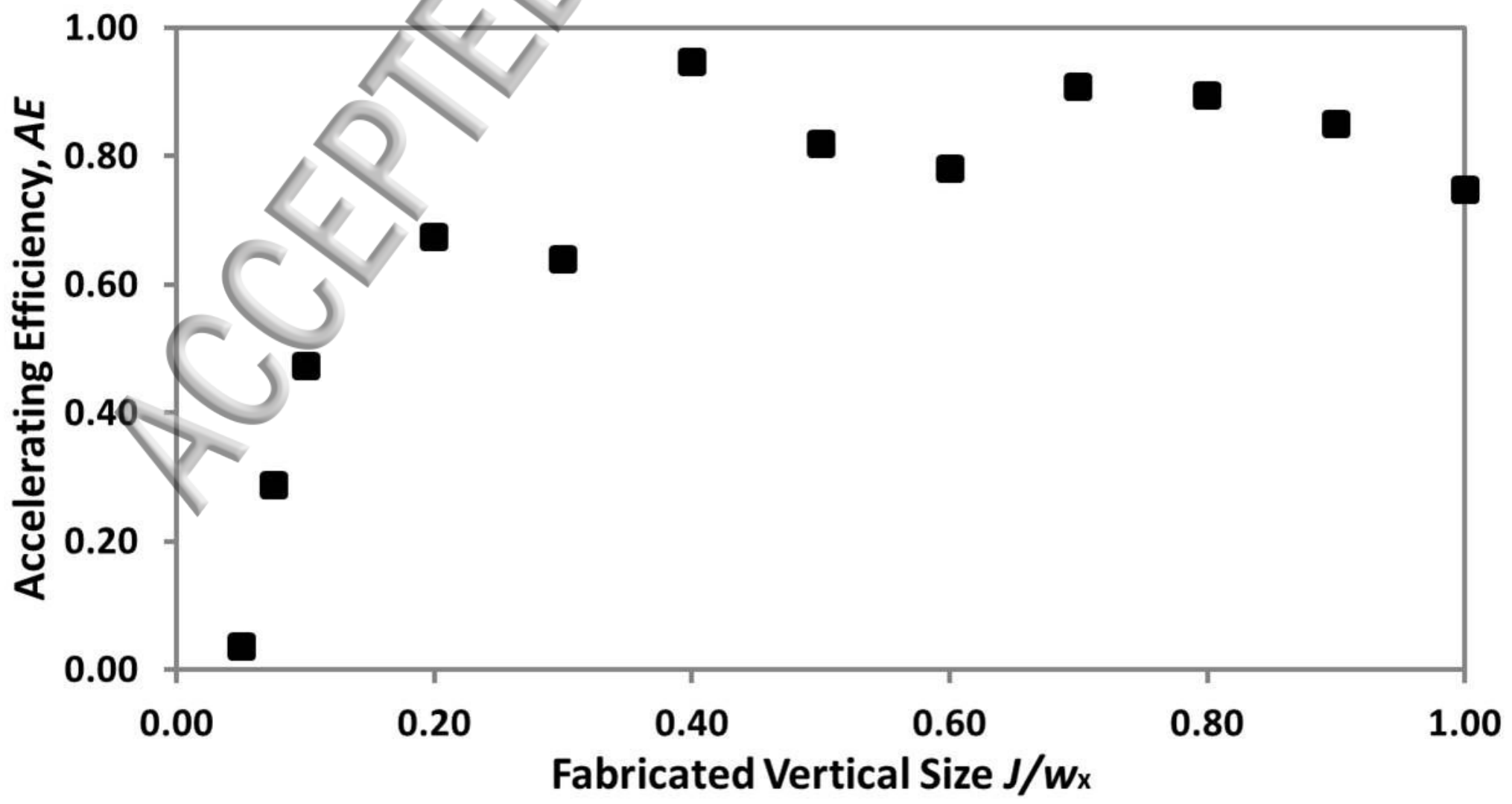

